\title{
Playful Interactions: A Critical Inquiry into Interactive Art and Play
}

\author{
Tine Bech \\ Digital Culture Research Centre \\ University of the West of England \\ UK \\ http://www.tinebech.com \\ mail@tinebech.com
}

Interactive art. Play theory. Sculpture. Public space. Playable city memory.

\section{BACKGROUND}

I am known for my public artworks that pop up in unexpected places. From bridges that light up as people pass over them, to live events where communities swim in clouds of Olympic colours, to battles on bikes, her works actively challenge our common assumptions about art, public space and participation. Using examples from her practice, Tine will share ideas on the relationship between play, interactive art, as well as play in the city, including the importance of the invitation, encouraging audiences to move from looking to doing, and the kinds of play that can be initiated through interactive artwork.

\section{SUMMARY}

Playable interactive art enables the creation of spaces and events that affords playful interactions and encounters - it invites people to be curious and seeks to engage audiences into dialogue and thereby opening up the possibility for play. I believe Play it is the call action within our emergent interactive environment. In fact the 'hallmark of play is that anyone can do it' (Bech 2012). Play is in the context of interactive art, doing - 'playing is doing' as Winnicott (1971) states. At the core of Winnicott's understanding of play is his focus on 'not so much the object used as the use of the object' (Winnicott 1971). This playing area is not 'inside' (as inner psychic reality), nor is it 'outside' (as in the separate to us world), in other words 'doing' is outside the individual but it is not in the external world - to interact with 'what is outside one has to do things, not simply to think or wish' - this is why play holds the potential to bridge the gap between doing and looking in the gallery. We will, if given the invitation, accept a call to engage in physical play with joy and delight. This distinct mode of embodied play holds the possibility for creating new ways of experiencing art.

As new technologies emerge different ways to creatively interact and collaborate emerge with them. There is a blurring of the boundaries between artists, audiences and participants that promotes new ways of interacting. I understand technologies by playing with them, testing them both in the studio and with audiences during exhibitions. Armitage (2011) writes that 'technology is not always a tool, an engineering substrate; it can be something to mould, to shape, to sculpt with'. Armitage's viewpoint is similar to the long tradition in visual art of exploring materials. Technologies have affordances and properties just like any material and, just as the grain of the wood allows me to make a particular kind of shape so it is by understanding the materiality of the technology that I can shape experiences; by working with the grain, or hacking it, to create art. There is no doubt that digital developments have provided new functionalities and aesthetic territories for artists to explore - fuelling the ongoing examination of what the art process can be about. In this view technology becomes animated - objects come to life and surveillance technology, motion sensor, and RFIDs are used to create artworks and develop participatory spaces. This sculptural approach to interactivity enables me to create playful interactions.

When people accept an invitation to engage in playful interaction they invest in the moment - they care. This interface allows participants to find new ways of engaging with people emotionally and socially. Playful interactive art hold the possibility to delight, tease and enchant audiences, to become players in the gallery, bridging the gap between looking and doing. This interaction is not about new technology but about creating art that moves people and allows them to find new ways of engaging, new ways of understanding and new ways of connecting. There are many social limitations in daily life that hold us back, but, once we accept the invitation to play, we engage and our surroundings once again become open to possibilities. Play has the ability to break down 
social barriers and, as such, it is transformative. After all, as we now know, play is the most pervasive behaviour across human cultures. Playful interaction in and around interactive artworks has the potential to become a meaningful form of interaction. The invitation is there!

\section{REFERENCES}

Armitage, T. (2001) Technology as a Material, Infovore, 22 August 2011.

http://infovore.org/archives/2011/08/22/technologyas-a-material/ (retrieved 25 June 2015).
Bech, T. (2012) Interactivity, Play and Audience Engagement. In Aceti, L., Jefferies, J., \& Papadimitriou, I. (eds.), Leonardo Electronic Almanac (Touch and Go), 18(3), 30-43. http://www.leoalmanac.org/vol18-no3-interactivityplay-and-audience-engagement/ (retrieved 25 June 2015).

Winnicott, D. W. (1971) Playing and Reality. Tavistoke Publications, Routledge. 